\title{
Wortstellung und Satztypmarkierung im Deutschen und im Ungarischen. Parallelen und Diskrepanzen ${ }^{1}$
}

\begin{abstract}
Das Deutsche und das Ungarische stellen für die kontrastive Grammatikforschung in der europäischen Linguistik insofern besonders interessante Vergleichssprachen dar, als sie einerseits genealogisch und typologisch große Unterschiede aufweisen, andererseits aber in den letzten Tausend Jahren durch das enge Zusammenleben im mitteleuropäischen Kulturraum auch auffällige Konvergenztendenzen zeigen, die mehrheitlich mit der unidirektionalen Wirkung des Deutschen auf das Ungarische zu erklären sind (vgl. Kiss 2003).

Im vorliegenden Beitrag werden nach einem überblicksartigen Vergleich relevanter Wortstellungstypen der beiden Sprachen besonders ausgewählte Interrogativsatztypen kontrastiert, die sowohl typologisch bedingte Diskrepanzen als auch bestimmte, auf einer abstrakten Ebene nachweisbare Parallelen aufweisen. Neben dem systematischen Vergleich werden exemplarisch auch mit Hilfe ausgewählter Korpusbelege veranschaulichte Performanzphänomene behandelt, um Konvergenzen im Sprachgebrauch zu zeigen.
\end{abstract}

\section{Problemstellung und Hypothesen}

In einschlägigen Grammatiken des Ungarischen (Tompa 1968, S. 337; Kiefer (Hg.) 1992, S. 83 ff.; Keszler/Lengyel 2008) wird die Wortstellung meistens als grammatisch weitgehend undeterminiert dargestellt, die grundsätzlich „,redefunktional“ geregelt und satztypunabhängig sei. Demgegenüber sei die deutsche Wortstellung in erster Linie durch die satztypenabhängige Stellung des Finitums determiniert (vgl. Altmann 1993a). Im folgenden Beispiel ist die Reihenfolge des Subjekts, des Objekts und des Verbs im ungarischen Satz frei variierbar, alle Kombinationen sind zugleich auch grammatisch korrekt. Im entsprechenden deutschen Satz ist die Wortstellung hingegen grammatisch geregelt und in erster Linie durch die zweite Stelle des finiten Verbs bestimmt.

Angefertigt mit der Unterstützung des Ungarischen Förderungsfonds Wissenschaftlicher Forschung (OTKA NN 79763) sowie des János-Bolyai-Forschungsstipendiums der Ungarischen Akademie der Wissenschaften. Für fruchtbare Diskussionen bedanke ich mich bei Prof. Dr. Péter Bassola. 
(1) Pista szereti Marit.

Pista liebt Maria-Akk

Auch möglich: Marit szereti Pista./Pista Marit szereti./Marit Pista szereti./Szereti Pista Marit./Szereti Marit Pista.

Stephan liebt Maria.

Im Entscheidungsinterrogativsatz sind im Ungarischen auch all diese Wortstellungen möglich, der interrogative Charakter wird nur durch die Frageintonation markiert. Im deutschen Entscheidungsinterrogativsatz ändert sich hingegen die Wortstellung: Liebt Stephan Maria?

Aus diesem Grund wird oft gemeint, dass die Wortstellung im Deutschen und im Ungarischen kaum vergleichbar sei. É.Kiss (2007) schreibt allerdings dem ungarischen Verb eine für die Serialisierung zentrale Rolle zu, indem das Verb den Satz in eine prä- und eine postverbale Satzhälfte teile, wobei erstere durch eine straffe Organisierung der Konstituenten gekennzeichnet werde, während in der zweiten Satzhälfte die Wortstellung frei variierbar sei.

Entgegen den herkömmlichen Auffassungen und teilweise in Anlehnung an É.Kiss (2007) möchte ich zeigen, dass die ungarische lineare Satzstruktur im Grunde durch den in der Satzmitte stehenden grammatisch geregelten Verbalkomplex bestimmt ist. In den Verbalkomplex können auch Konstituenten integriert werden, die im Deutschen nicht als Verbalkomplexteile angesehen werden. Die Tatsache, dass die Serialisierung in beiden Sprachen grundsätzlich durch die Stelle des Verbalkomplexes determiniert ist, stellt trotz aller Einzelunterschiede eine wesentliche Ähnlichkeit dar, die einen systematischen Vergleich der Serialisierungstypen der beiden Sprachen erlaubt. Der Verbalkomplex teilt den ungarischen Satz in eine vordere und eine hintere Satzhälfte, wobei die vordere Satzhälfte trotz relevanter Unterschiede mit dem deutschen Vorfeld zumindest funktional vergleichbar ist. ${ }^{2}$

Ferner möchte ich auch zeigen, dass die deutsche und die ungarische Wortstellung auch unter dem Aspekt ihrer Satztypspezifität durchaus vergleichbar sind.

Wenn man davon ausgeht, dass das System der deutschen Satztypen in erster Linie auf der Verb-zweit-/Verb-erst-Opposition basiere, kann man für das Ungarische eine gewissermaßen ähnliche Opposition postulieren, abhängig davon, ob die vor dem Verbalkomplex stehende vordere Satzhälfte realisiert wird oder nicht. Die VK2-/VK1-Opposition ${ }^{3}$ ist im Ungarischen nicht so fest an Satztypen gebunden wie die erwähnte deutsche Opposition, weist jedoch - wie gezeigt wird - ziemlich starke satztypspezifische Tendenzen auf. Vorläufig wird dies mit einem vereinfachten Beispiel veranschaulicht:

2 Vgl. auf der deutschen Seite vor allem Zifonun/Hoffmann/Strecker (1997, Bd. 2, S. 1495 ff.) sowie unter historischem Aspekt Admoni (1990, S. 69 ff.), auf der ungarischen Seite Kenesei/Vago/Fenyvesi (1998, S. 15 f.).

$\mathrm{VK}=$ Verbalkomplex. 


$\begin{array}{llll}\text { Ebben a boltban lehet zöldséget } & \text { kapni. } \\ \text { dies-in DET Laden-in könn-3SG Gemüse-AKK } & \text { kaufen } \\ \text {,In diesem Laden kann man Gemüse kaufen.‘ } & \\ \text { Lehet ebben a boltban zöldséget } & \text { kapni? } \\ \text { könn-3SG dies-in DET Laden-in Gemüse-AKK kaufen } \\ \text { "Kann man in diesem Laden Gemüse kaufen?‘ }\end{array}$

In beiden Sätzen unter dem Beispiel (3), also sowohl im Deklarativ- als auch im Interrogativsatz, sind beide aufgeführten Wortstellungen grammatisch korrekt. In einem neutralen Verwendungskontext wird jedoch der Deklarativsatz meistens mit einer als Topik funktionierenden Nominal- oder Adverbialphrase eingeleitet, während Interrogativsätze mehrheitlich mit dem Verbalkomplex anfangen.

Die Hypothesen für die vorliegende Arbeit können also in folgenden Punkten zusammengefasst werden.

1) Für die Beschreibung der ungarischen Wortstellung lässt sich ein Modell entwickeln, in dessen Mittelpunkt die Kategorie des Verbalkomplexes steht. Dadurch können die deutsche und die ungarische Wortstellung miteinander verglichen werden.

2) Die ungarische erste Satzhälfte vor dem Verbalkomplex weist sowohl hinsichtlich ihrer pragmatischen Funktionen als auch hinsichtlich ihrer Satztypspezifität gewisse Analogien zum deutschen Vorfeld auf.

3) Die ungarische Wortstellung ist jedoch trotz dieser Ähnlichkeiten grammatisch insgesamt weniger determiniert als die deutsche und ermöglicht in allen Satztypen feinere pragmatische Differenzierungen hinsichtlich der Informationsstrukturierung. Diese Differenzierungen werden im Deutschen mit verschiedenen Mitteln auf verschiedenen Ebenen des Sprachsystems realisiert.

Methodisch wird zuerst unter dem Einfluss der einschlägigen germanistischen Forschungen ein Modell für das Ungarische entwickelt. Nachher wird aber wieder auf das Deutsche Bezug genommen. Der Sprachvergleich ermöglicht eine fein differenzierte Analyse und neue Erkenntnisse auf der Seite beider kontrastierter Sprachen.

\section{Die lineare Satzstruktur im Ungarischen}

Nach den Hypothesen wird zuerst gezeigt, dass die ungarische Wortstellung trotz wesentlicher Unterschiede mit dem Deutschen vergleichbare grammatische Restriktionen aufweist.

Das Urungarische war vermutlich eine SOV-Sprache, die etwa gegen Ende der Wanderungszeit eine grammatische Auflockerung und einen Übergang zur einer im Grunde pragmatisch determinierten Wortstellung aufgrund des Grundmusters Topik - Fokus - Verb - XP erlebte (vgl. Kiss/ 
Pusztai 2003, S. 253, 483 ff.). Die weiteren Entwicklungen, besonders die Entstehung des Verbpartikelsystems, zogen jedoch erneut eine gewisse grammatische Verfestigung nach sich. Ungarische Verbpartikeln drücken teilweise Aspektualität aus, teilweise differenzieren sie den deutschen Verbpartikeln ähnlich die Verbbedeutung. In präverbaler Position werden sie mit dem Verb zusammengeschrieben und tragen den Akzent des Verbs, in postverbaler Position sind sie getrennt geschrieben und nicht akzentuiert.

$$
\begin{aligned}
& \text { ir / megir / Nem irtmeg semmit. } \\
& \text {,schreiben' ,fertigschreiben }{ }^{64} \text {,Er/sie schrieb nichts fertig. }
\end{aligned}
$$

$$
\begin{array}{ll}
\text { megy / elmegy / Nem ment el. } \\
\text {,gehen` ,weggehen` ,Er/sie ging nicht weg.“ }
\end{array}
$$

Nach der am weitesten verbreiteten Auffassung über die ungarische Serialisierung wird für den ungarischen Satz eine im Grunde auf dem GB-Modell basierende Phrasenstruktur postuliert, allerdings mit einer ungarischen Besonderheit, indem die Phrasentypen durch ihre pragmatische Funktion bestimmt seien (vgl. Kiefer (Hg.) 1992, S. 87 ff.; É.Kiss 2002).

(6) Phrasenstruktur des ungarischen Satzes (nach É.Kiss 2002)

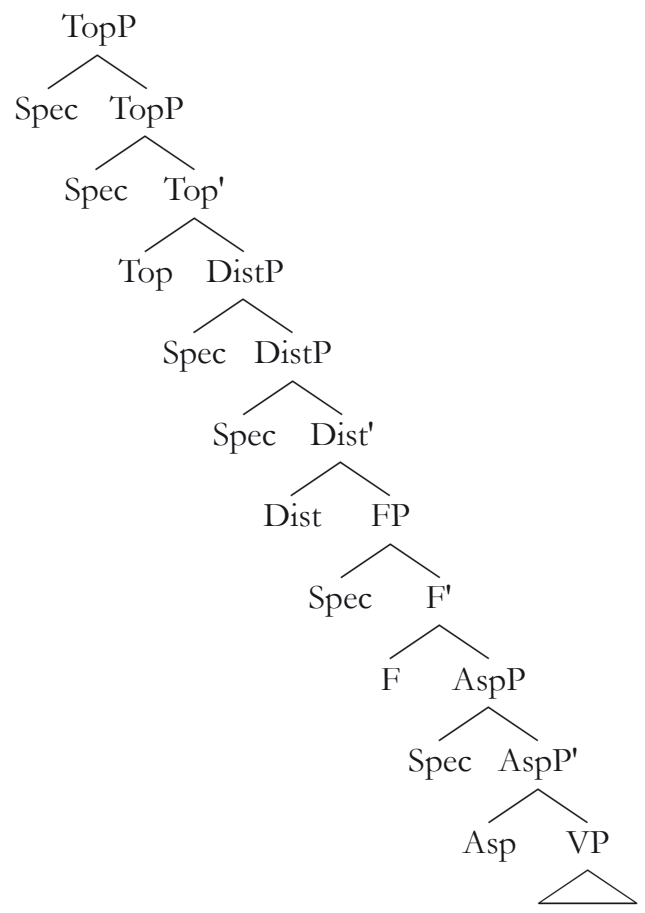

Die verwendungsneutrale Zitierform der ungarischen Verben ist die Form 3SG und nicht der Infinitiv wie im Deutschen. 
Nach É.Kiss (2002) stehen eventuell auch mehrere Topikphrasen am Satzanfang, zwischen dem Topik und dem Fokus könnten die distributionellen Quantoren stehen, präverbal stehe die Fokusphrase oder die als Spezifizierer der so genannten Aspektphrase dienende Verbpartikel, wobei die beiden Positionen einander ausschließen würden. Hinter dem Verb stünden Erweiterungen der Verbalphrase, die weder mit Topik- noch mit Fokusmerkmal versehen seien, höchstens könne man in diesem Bereich über Fokusprojektion sprechen. Nach dieser, durch die angelsächsische Forschungstradition beeinflussten Theorie bestehe die „Eigenart“ der ungarischen Wortstellung in der im Grunde pragmatischen Determinierung der linearen Abfolge, die sich besonders in der präverbalen Fokusposition manifestiere. In dieser Position korreliere die markierte syntaktische Stellung einer nicht verbalen Konstituente auf der phonologischen Ebene mit dem Satzakzent bzw. auf der pragmatischen Ebene mit dem Fokusmerkmal und determiniere zugleich die invertierte Stellung der Verbpartikel.

Im vorliegenden Phrasenstrukturbaum wird jedoch übersehen, dass die Korrelation zwischen der präverbalen Phrase mit dem Satzakzent, mit dem Fokusmerkmal sowie mit der invertierten Position der Verbpartikel empirisch keineswegs vollständig nachzuweisen ist. Erstens steht die Interrogativphrase unabhängig von den realen Hintergrund-Fokus-Verhältnissen obligatorisch in der markierten präverbalen Position, was bei É.Kiss (2002) mit dem inhärenten Fokusmerkmal der Interrogativphrase erklärt wird. Demgegenüber meine ich, dass die Feststellungen von Altmann (1993b) in Bezug auf die Hintergrund-Fokus-Gliederung des Ergänzungsinterrogativsatzes, dass nämlich auch in diesem Satztyp je nach Kontext verschiedene Phrasen fokussiert werden können und sogar Fokusprojektion möglich ist, nicht nur für das Deutsche stimmen. Auch im Ungarischen trägt die Interrogativphrase nicht unbedingt einen Fokusakzent. ${ }^{5}$

- A jobb kezemben egy almát tartok.
DET recht Hand-POSS-1SG-in ein Apfel-AKK halt-1SG
In meiner rechten Hand halte ich einen Apfel.‘
- Es mit tartasz a BAL kezedben?
Und was-AKK halt-2SG DET link Hand-POSS-2SG-in
,Und was hältst du in deiner LINKEN Hand?c

Hinzu kommt, dass in emphatischen Realisierungen sowohl die Interrogativphrase als auch die Verbpartikel präverbal stehen. ${ }^{6}$

Am eindeutigsten sind diejenigen Beispiele, in denen eine andere Phrase mit einem kontrastiven Akzent versehen ist. In kontextgebundenen Sätzen kann jedoch nach meinem Sprachgefühl eine andere Phrase auch einen neutralen Satzakzent tragen.

6 Damit könnten Stellungsregularitäten innerhalb des Verbalkomplexes als Grundlage für die Abgrenzung des Interrogativsatzes und des Exklamativsatzes dienen. 
Mit meg nem tett a gyerekeiért!

Was-AKK VPART NEG tun-PAST-3SG DET Kind-PL-POSS-3SG-für ,Was alles hat er/sie nicht für seine/ihre Kinder getan!

Zweitens stehen auch Satzoperatoren, nämlich die Negationspartikel nem/ne (bei Satznegation) sowie die Imperativpartikel hadd obligatorisch präverbal. Als Partikeln können sie selbst nicht fokussiert werden. Vielmehr werden sie in den Verbalkomplex integriert und verschmelzen mit dem Finitum zu einem phonetischen Wort:

Klaus NEM olvasta a levelet.

Klaus NEG les-PAST-3SG DET Brief-AKK

,Klaus hat den Brief nicht gelesen.

$\begin{array}{llll}\text { HADD olvassa } & \text { Klaus a levelet. } \\ \text { IMPPART les-IMP-3SG Klaus } & \text { DET } & \text { Brief-AKK } \\ \text {,Lasst Klaus den Brief lesen!‘ } & & \end{array}$

Die Negationspartikel steht in emphatischen Aussagen sogar zwischen Verbpartikel und Finitum, also in der Mitte des Verbalkomplexes.

El nem olvasná semmi pénzért!.
VPART NEG les-KOND-3SG nichts
„Er würde das um keinen Preis lesen!

Drittens stellen die so genannten distributionellen Quantoren sowie die Phrasen mit der Fokuspartikel is ein besonderes Problem dar. Wenn sie präverbal stehen und auch den Fokusakzent tragen, wird dadurch keine invertierte Stellung der Verbpartikel determiniert.
Tegnap MINdenki megfürdött.
Gestern alle(SG) VPART-bad-PAST-3SG
,Gestern haben alle gebadet.
Tegnap KLAUS is
megfürdött.
Gestern Klaus auch VPART-bad-PAST-3SG
,Gestern hat auch Klaus gebadet.

Sie können ferner auch vor der präverbalen Position stehen, aber auch in diesem Fall können sie einen Fokusakzent tragen.
Tegnap MINdenki a
tóban fürdött meg. ,Gestern haben alle im See gebadet.

$\begin{array}{lll}\text { Tegnap KLAUS is a tóban fürdött } & \text { meg. } \\ \text { Gestern Klaus auch DET See-in bad-PAST-3SG } & \text { VPART } \\ \text {,Gestern hat auch Klaus im See gebadet. } & \end{array}$


Die Phrasen mit is ,auch“ schließt É.Kiss (2002) von den Fokusphrasen aus, weil sie keine exhaustive, sondern eine inklusive Interpretation haben. Dagegen spricht jedoch die Beobachtung, dass lexikalische Paraphrasen für die Partikel is wie z.B. többek kö̋ött unter Anderem` eine gewöhnliche Fokusposition mit Inversion der Verbpartikel determinieren. ${ }^{7}$ Das besondere syntaktische Verhalten der vorliegenden Phrasentypen erkläre ich damit, dass sie ihr Fokusmerkmal auf die danach stehende Position projizieren. Wenn sie präverbal stehen, wird zugleich auch der ganze Verbalkomplex mitfokussiert. Bei der Fokuspartikel is hängt es wohl auch mit ihrem etymologischen Zusammenhang mit der koordinierenden Konjunktion és ,und' zusammen, wodurch sie zugleich die enge Verbindung mit der nachfolgenden Phrase herstellt.

Ich gehe davon aus, dass im Mittelpunkt eines adäquaten Modells der ungarischen Wortstellung der Verbalkomplex steht, der einen präverbalen Teil, das eigentliche Verbum finitum sowie einen postverbalen Teil umfasst. ${ }^{8}$ Die präverbale Position wird in den Verbalkomplex integriert. Die Reihenfolgeverhältnisse im Verbalkomplex sind nach einer strengen grammatischen Hierarchie geregelt, die in der folgenden Tabelle dargestellt wird. ${ }^{9}$

Der Verbalkomplex im Ungarischen

\begin{tabular}{|c|c|c|}
\hline $\begin{array}{c}\text { PRÄVERBALER } \\
\text { TEIL }\end{array}$ & \multirow{11}{*}{$\begin{array}{l}\text { VERBUM } \\
\text { FINITUM }\end{array}$} & $\begin{array}{c}\text { POSTVERBALER } \\
\text { TEIL }\end{array}$ \\
\hline VPART & & \\
\hline Interrogativphrase & & VPART \\
\hline Interrogativphrase + VPART (emphatisch) & & \\
\hline Satznegator & & VPART \\
\hline VPART + Satznegator (emphatisch) & & \\
\hline Imperativpartikel hadd & & VPART \\
\hline Imperativpartikel hadd + Negator & & VPART \\
\hline $\mathrm{XP}-i s /$ Dist.Q + VPART & & \\
\hline $\mathrm{XP}-i s /$ Dist.Q + Fokusphrase & & VPART \\
\hline Fokusphrase & & VPART \\
\hline
\end{tabular}

Wenn jedoch többek között unter Anderem' und is ,auch' kombiniert werden, was durchaus möglich ist, liegt keine invertierte Wortstellung vor. Die Erklärung dieser Wortstellungsregularitäten bedarf weiterer Untersuchungen.

8 Nicht berücksichtigt werden hier Erweiterungen des Verbalkomplexes mit Auxiliar- und Modalverben, deren Stellung besonderen grammatischen Regeln unterworfen ist.

$9 \quad$ Die vorliegende Tabelle kann aus Umfangsgünden nicht alle Wortstellungstypen innerhalb des Verbalkomplexes enthalten. Unberücksichtigt bleiben auch Strukturen mit nominalem Prä- 
Ein wesentliches empirisches Argument für meine Annahme besteht in der phonetischen Integration der präverbalen Position mit dem Verbalkomplex. Nicht nur eine präverbal stehende Verbpartikel, sondern auch die präverbalen Operatoren und die präverbalen Fokusphrasen bilden mit dem Verb eine Intonationsphrase. Von der syntaktischen Integration zeugt die Tatsache, dass zwischen der präverbalen Position und dem Verb auf keinen Fall eine Parenthese stehen kann.

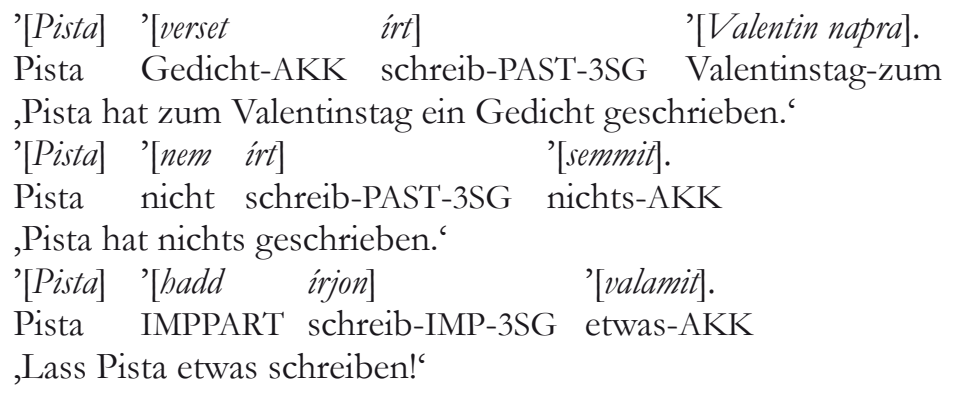

Die neutrale Serialisierung des ungarischen Satzes ist somit durch den in der Mitte stehenden Verbalkomplex in eine vordere und eine hintere Satzhälfte gegliedert, was im Grunde auf der Unterscheidung Hintergrund/ Vordergrund beruht. Die vordere Satzhälfte ist einerseits für topikalisierte Ausdrücke, andererseits auch für andere Hintergrundelemente, für textkonnektive, modale und evidenziale Ausdrücke reserviert. Ein wesentlicher Unterschied zum Vorfeld des deutschen Satzes besteht in der Möglichkeit der Mehrfachbesetzung dieser Satzhälfte durch mehrere autonome Phrasen. In markierten Strukturen kann diese Hintergrund-Vordergrund-Struktur ebenso wie im Deutschen verletzt werden und auch Informationen im Vordergrund können in der vorderen Satzhälfte vorkommen. Die hintere Satzhälfte ist für Fokusprojektionen bzw. für sonstige Vordergrundelemente reserviert. Textkonnektive Elemente sind hier nicht möglich, modale und evidenziale Merkmale nur in markierten Strukturen, in denen sie eigentlich als parenthetische Nachträge wirken.

dikat. Ferner muss auch bemerkt werden, dass die Integration des präverbalen Teils in den einzelnen Fällen unterschiedlich stark ist. Bei den distributionellen Quantoren sowie im Falle der Phrasen mit is ist die phonetische Integration deutlich schwächer als in den anderen Fällen. Im Falle der Kombination von Fokusphrase+Verb müssen wohl auch unterschiedliche Stufen der Integration unterschieden werden (bis hin zur Inkorporation), bei denen auch der Artikelgebrauch in der Fokusphrase entscheidend sein kann. Fokusphrasen in artikellosen Fügungen werden bei É.Kiss/Kiefer/Siptár (1998) ähnlich wie die Verbpartikeln als „Verbmodifizierer" angesehen. Eine feinere Analyse dieser Frage bedarf weiterer Untersuchungen und würde den Rahmen des vorliegenden Beitrags sprengen. 
(18) Gesamtmodell der ungarischen Wortstellung

\begin{tabular}{|c|c|c|c|c|}
\hline \multirow{2}{*}{$\begin{array}{l}\text { Vordere } \\
\text { Satzhälfte }\end{array}$} & \multicolumn{3}{|c|}{ Verbalkomplex } & \multirow{2}{*}{$\begin{array}{c}\text { Hintere } \\
\text { Satzhälfte }\end{array}$} \\
\hline & \multirow{4}{*}{$\begin{array}{l}\text { präverba- } \\
\text { ler Teil }\end{array}$} & \multirow{4}{*}{$\begin{array}{l}\text { Verbum } \\
\text { finitum }\end{array}$} & \multirow{4}{*}{$\begin{array}{l}\text { postverba- } \\
\text { ler Teil }\end{array}$} & \\
\hline Topik & & & & \multirow{3}{*}{$\begin{array}{l}\text { Vordergründige } \\
\text { Komplemente und } \\
\text { Supplemente }\end{array}$} \\
\hline $\begin{array}{l}\text { andere Hinter- } \\
\text { grundelemente }\end{array}$ & & & & \\
\hline $\begin{array}{c}\text { textkonnektive, } \\
\text { modale, evidenziale } \\
\text { Marker }\end{array}$ & & & & \\
\hline HINTERGRUND & \multicolumn{4}{|c|}{ VORDERGRUND } \\
\hline
\end{tabular}

\section{Syntaktische Parallelen und Diskrepanzen in der ungarischen und deutschen Wortstellung}

\subsection{Vorfeldbesetzung im Deutschen bzw. die Besetzung der ersten Satzhälfte im Ungarischen}

Die Verb-zweit/Verb-erst-Opposition, die auf irgendeine Weise in vielen europäischen Sprachen zu beobachten ist, ist in den germanischen Sprachen am stärksten grammatisch geregelt und wurde zu einem Merkmal der Deklarativ-Interrogativ-Unterscheidung (19). In der deutschen Gegenwartssprache kann man besonders im lockeren, weniger durch die schriftsprachliche Norm beeinflussten Sprachgebrauch bestimmte Auflockerungstendenzen beobachten wie den bei Önnerfors (1997) als eigenständigen Strukturtyp ausgewiesenen, in narrativen, mehrheitlich lockeren gesprochenen Texten wie z.B. in Witzen benutzten so genannten V1-Deklarativsatz (20).

Klaus kommt./Es regnet. vs. Kommt Klaus?/Regnet es?

(20) Kam ein Mann in die Kneipe und sagte ... (vgl. Önnerfors 1997)

Im Ungarischen sind VK1-Deklarativsätze praktisch uneingeschränkt möglich. Sie stellen eine so genannte, thetische Perspektive' dar, in denen das Ereignis als einheitlich und ungegliedert dargestellt wird. Das Beispiel (21) fängt mit dem Finitum, (22) mit der in den Verbalkomplex integrierten Fokusexponente an. Im Deutschen stellen thetische Sätze in Texten, die enger an die schriftsprachliche Norm gebunden sind, eine V2-Struktur dar, z.B. mit einem nicht-phorischen es oder da oder mit einer intonatorisch als Fokusexponente markierten Phrase im Vorfeld (23)-(24). 


\author{
(21) Jön a tavas\% (VK1 (V1) - thetisch) \\ komm-3SG DET Frühling \\ ,Es kommt der Frühling. \\ (22) Virág nyilik a kertben. (VK1 (FP-V) - thetisch) \\ Blume blüht DET Garten-in \\ ,Blumen blühen im Garten.‘ \\ (23) Es kommt der Frübling./Da kommt jemand. \\ (24) Der FRÜHling kommt. (Thetische V2-Deklarativsätze)
}

Im Falle des Deklarativsatzes kann also für beide Sprachen festgehalten werden, dass die V1-Struktur (bzw. im Ungarischen die VK1-Struktur) markiert ist und die thetische Perspektive realisiert. Im Deutschen ist sie allerdings viel beschränkter zu benutzen als im Ungarischen. Im Entscheidungsinterrogativsatz ist hingegen die V1-Stellung (im Ungarischen die VK1-Stellung) die unmarkierte. Auch wenn im Ungarischen Entscheidungsinterrogativsätze durchaus möglich sind, zeigen die Beispiele (25)-(27), dass die neutralen, unmarkierten Entscheidungsinterrogativsätze auch im Ungarischen eigentlich mit dem Verbalkomplex anfangen. Der Unterschied zum Deutschen besteht besonders darin, dass eine VK1-Struktur im Ungarischen nicht nur mit dem Finitum anfangen kann wie in (25), sondern auch mit sämtlichen Konstituenten, die - wie bisher gezeigt - in der präverbalen, in den Verbalkomplex integrierten Position stehen, unter Anderem mit der Verbpartikel (26) bzw. mit der präverbalen Fokusexponente (27). ${ }^{10}$

Hallottátok az uij bireket?
hör-PAST-2PL DET neu Nachricht-PL-AKK
,Habt ihr die neuen Nachrichten gehört??

Megfó"zted már az ebédet?
VPART-koch-PAST-2SG schon DET Mittagessen-AKK
„Hast du schon das Mittagessen gekocht?

$\begin{array}{llll}\text { Víz ment } & a & \text { szemedbe? } \\ \text { Wasser } & \text { geh-PAST-3SG } & \text { DET Auge-POSS-2SG-in } \\ \text {,Ist Wasser in Deine Augen gekommen? }\end{array}$

3.2 Fallbeispiel: Korpusuntersuchung zu den deutschen und ungarischen Wortstellungstypen in Entscheidungsinterrogativsätzen

Der folgende Vergleich basiert auf der systematischen Auswertung der Daten eines deutsch-ungarischen Vergleichskorpus. Damit will ich einerseits

${ }_{10}$ Zu den Beispielen unter (25)-(27) vgl. auch Komlósy (1992, S. 500) bzw. Bassola (2001, S. 31). 
die bisherigen Thesen mit Hilfe von Korpusdaten validieren, andererseits ist die Korpusbefragung zur Ermittlung der Funktion markierter und stark situationsgebundener Nebentypen unentbehrlich.

Das zugrunde liegende, selbst erstellte Korpus enthält im Falle beider Sprachen mehr als 4 Millionen Textwörter. ${ }^{11}$ Die Erstellung des Korpus war durch die gegebenen Möglichkeiten ziemlich beschränkt. Das Grundkonzept bestand darin, für beide Sprachen ähnliche Texte in etwa ähnlichem Umfang aufzunehmen, die in schriftlich fixierter bzw. elektronisch suchbarer Form vorliegen, aber - auch wenn in unterschiedlichem Maße - Merkmale der konzeptionellen Mündlichkeit (im Sinne von Koch/Österreicher 1985 bzw. Ágel/Hennig 2006) enthalten. Die Mehrheit der Texte sind Bundestags- bzw. ungarische Parlamentsprotokolle, in denen Debatten und Zwischenrufe unterschieden werden. Die Texte in den Debatten sind meistens vorbereitete, relativ stark an die schriftsprachliche Norm gebundene Reden, während die Zwischenrufe, die vom Protokollführer auch markiert werden, spontane, unvorbereitete Äußerungen darstellen. In bescheidenem Umfang konnte ich für beide Sprachen auch transkribierte Gespräche anschaffen, die in einem Schüler- bzw. in einem Studentenheim aufgenommen wurden. ${ }^{12}$ Ferner wurden auch Dramentexte aus der ersten Hälfte des 20. Jahrhunderts ausgewertet. ${ }^{13}$ In allen Teilkorpora wurden nach dem Zufallsprinzip 500 Entscheidungsinterrogativsätze ausgewählt bzw. es wurden in den kleineren Korpora, wo so viele nicht vorhanden waren, alle auffindbaren Belege berücksichtigt. Die Suche erfolgte mit Hilfe des in allen Teilkorpora ziemlich zuverlässig benutzten satzabschließenden Fragezeichens, eventuelle Fehlbelege wurden manuell gestrichen.

Die Häufigkeitsverteilung der V1- und der V2-Typen im Deutschen bzw. der VK1- und VK2-Typen im Ungarischen ${ }^{14}$ weist in den Teilkorpora, die einen in hohem Maße situationsgebundenen und teilweise auch lockeren Sprachgebrauch repräsentieren, große Ähnlichkeiten auf. In Bundestagsbzw. Parlamentsdebatten, in denen sich der Sprachgebrauch strenger nach der schriftsprachlichen Norm richtet, ergibt sich aber ein hochsignifikanter

11 Zu genauen Umfangsangaben sowie zur Struktur der Teilkorpora siehe den Anhang.

12 Die deutschen Gespräche stammen vom Ende der 90er Jahre, die ungarischen aus den 70er Jahren. Die Markierungstechniken der grundlegenden Satzmodi verändern sich aber vorhersagbar nicht so schnell. Auch nach meinem Sprachgefühl könnte man ähnliche Belege auch in zeitgenössischen Gesprächen finden.

13 Auf der deutschen Seite Brecht, Dürrenmatt, Grass und Ödön von Horváth, auf der ungarischen Gyula Illyés, Áron Tamási und László Németh. Modernere Texte waren leider aus urheberrechtlichen Gründen in elektronisch suchbarer Form nicht auffindbar. Auch diese Texte können aber einen gewissen Anhaltspunkt zu den vorliegenden Untersuchungen liefern.

14 Im Deutschen gibt es darüber hinaus auch Verb-letzt-Strukturen, die jedoch die Ergebnisse nicht entscheidend beeinflussen. Eingliedrige Kurzsätze bilden nicht den Gegenstand des vorliegenden Beitrags, sie mussten nur insofern berücksichtigt werden, als sie auf die Häufigkeitsverteilung der anderen Satztypen zurückwirken. 
Unterschied. Diese Häufigkeitsdaten weisen aller Wahrscheinlichkeit nach darauf hin, dass während V2-Entscheidungsinterrogativsätze im Deutschen bzw. VK2-Entscheidungsinterrogativsätze im Ungarischen hinsichtlich ihrer pragmatischen Funktion Ähnlichkeiten aufweisen, sie auf der Systemebene doch einen unterschiedlichen Status haben.

(28) Häufigkeitsverteilung der Wortstellungstypen in deutschen und ungarischen Entscheidungsinterrogativsätzen

\begin{tabular}{|c|c|c|c|c|c|c|c|c|}
\hline & \multicolumn{4}{|c|}{ DEUTSCH } & \multicolumn{3}{|c|}{ UNGARISCH } \\
\cline { 2 - 9 } & $\begin{array}{c}\text { Bundes- } \\
\text { tagsde- } \\
\text { batten }\end{array}$ & $\begin{array}{c}\text { Zwi- } \\
\text { schen- } \\
\text { rufe }\end{array}$ & $\begin{array}{c}\text { Dra- } \\
\text { men }\end{array}$ & $\begin{array}{c}\text { Ge- } \\
\text { spräche }\end{array}$ & $\begin{array}{c}\text { Parla- } \\
\text { ments- } \\
\text { debat- } \\
\text { ten }\end{array}$ & $\begin{array}{c}\text { Zwi- } \\
\text { schen- } \\
\text { rufe }\end{array}$ & $\begin{array}{c}\text { Dra- } \\
\text { men }\end{array}$ & $\begin{array}{c}\text { Ge- } \\
\text { spräche }\end{array}$ \\
\hline V1/VK1 & $93,4 \%$ & $59,9 \%$ & $47,6 \%$ & $46,2 \%$ & $66,8 \%$ & $56,3 \%$ & $53,2 \%$ & $42,7 \%$ \\
\hline V2/VK2 & $2,8 \%$ & $14,7 \%$ & $19,8 \%$ & $26,0 \%$ & $24,2 \%$ & $17,5 \%$ & $10,8 \%$ & $23,3 \%$ \\
\hline KURZ & $3,4 \%$ & $24,4 \%$ & $31,0 \%$ & $26,0 \%$ & $9,0 \%$ & $26,2 \%$ & $36,0 \%$ & $34,0 \%$ \\
\hline V-letzt & $0,4 \%$ & $1,0 \%$ & $1,6 \%$ & $1,8 \%$ & & & & \\
\hline
\end{tabular}

\section{VK2-Entscheidungsinterrogativsätze im Ungarischen}

Wenn das Vorfeld bzw. die vordere Satzhälfte im Entscheidungsinterrogativsatz besetzt wird, können dadurch besondere pragmatische Funktionen realisiert werden. Im Ungarischen stehen in der vorderen Satzhälfte häufig textverknüpfende Elemente, Adverbien, Pronominaladverbien, anaphorische sowie auch Anredepronomina, die einen unmittelbaren Situationsbezug herstellen (29), ferner auch modale und evidenziale Marker wie z.B. die deliberative, eine nachdenkende Frage markierende Modalpartikel vajon (30) sowie evidenziale Ausdrücke wie Ön szerint, Ihrer Meinung nach (31), usw. Ferner können auch satzwertige Konstruktionen in der ersten Satzhälfte stehen (29’).

(29) Addig maradjak fönn, vagy menjeke le? [Debatten] Bis dahin bleib-IMP-1SG oben oder geh-IMP-1SG runter ,Soll ich bis dahin hier oben bleiben, oder runter gehen?‘

(29’) Amig itt vagy, maradjak. fönn, vagy menjek. le?

Solange hier sein-2SG

,Solange du hier bist, soll ich hier oben bleiben, oder runter gehen?

Vajon az ellenzék tehet arról? [Debatten]

PART DET Opposition tun-KOND-3SG darüber

,Ob etwa die Opposition daran schuldig ist ...? 


$\begin{array}{llll}\text { Ön szerint kulturáltan } & \text { jártak } & \text { el } & a \\ \text { Sie gemäß zivilisiert } & \text { verfahr-PAST-3PL } & \text { VPART } & \text { DET } \\ \text { rendöröke? [Debatten] } & & & \\ \text { Polizisten } & \end{array}$

„Haben die Polizisten Ihrer Meinung nach zivilisiert verfahren?

Am interessantesten sind jedoch Beispiele mit einer als Topik zu interpretierenden referierenden Phrase am Satzanfang. Das Topik des Interrogativsatzes steht außerhalb der Skopusdomäne des Interrogativoperators, wird selber nicht in Frage gestellt, sondern eigentlich vor der Fragestellung als Grundlage für die Frage festgehalten. Es stellt die gemeinsame Basis von Sprecher und Hörer, eine Art common ground der Fragestellung dar.
$\begin{array}{llllll}\text { A } & \text { kormány } & \text { nem } & \text { vette észre } & \text { ezt } & a \\ \text { DET } & \text { Regierung } & \text { nicht } & \text { bemerk-PAST-3SG } & \text { dies-AKK } & \text { DET }\end{array}$ nyilvánvaló megtévesztést?
offensichtlich Täuschung-Akk
„Die Regierung - hat sie diese offensichtliche Täuschung nicht be- merkt?' [Debatten]

Az 1999-ben elkö̈nyelt biány véletlenül nem a belga
DET 1999-in gebucht Defizit zufällig nicht DET belgisch
KBC-Bank offshore-cégének.
KBC-Bank Offshore-Firma-POSSESSOR
bankszámláján landolt?
Bankkonto-POSSESSUM-auf land-PAST-3SG
,Das 1999 gebuchte Defizit - ist es zufällig nicht auf dem Bankkonto
der Offshore-Firma der belgischen KFC-Bank gelandet?‘ [Debatten]

Im Beispiel (32) wird in der Frage eines oppositionellen Abgeordneten am Anfang die in einer Parlamentsdebatte selbstverständliche Information festgehalten, dass diese Frage in Bezug auf die Regierung gestellt wird. Vor diesem common ground wird gefragt, ob die Regierung diese offensichtliche Täuschung nicht bemerkt hat. Im Beispiel (33) wird auf das Defizit hingewiesen und vor diesem Hintergrund wird die Frage nach seinen Ursachen bzw. nach dem Schicksal des fehlenden Geldbetrags gestellt.

Diese Funktion, die Topikalisierung einer Konstituente im Interrogativsatz, und zwar als gemeinsamer Hintergrund, lässt sich in den meisten europäischen Sprachen sehr schwierig paraphrasieren. Eine Möglichkeit bieten in vielen Sprachen Herausstellungs- bzw. Cleft-Strukturen, in denen die extraponierten Phrasen als Topik betrachtet werden können. Doch, wie Altmann (2009, S. 22) bemerkt, sind sie zugleich auch fokal, weil extraponierte Phrasen notwendigerweise stets auch intonatorisch abgegrenzt werden und einen eigenen Akzent bekommen. 
War es nicht die Weltanschaunng, die Bio erst zu Bio machte? (Der Spiegel, zitiert nach Altmann 2009, S. 22)

Die in der vorderen Satzhälfte des ungarischen Entscheidungsinterrogativsatzes stehende Topikphrase ist demgegenüber nicht fokal, sondern bildet gerade den gemeinsamen Hintergrund. Da Herausstellung auch im Ungarischen möglich ist, kann der Unterschied am besten an den beiden ungarischen Beispielen mit und ohne extraponierte erste Konstituente demonstriert werden. Die extraponierte, auch intonatorisch vom Restsatz abgegrenzte Phrase verfügt über einen selbstständigen Fokusakzent, unabhängig von den Akzentverhältnissen des Restsatzes. Das durch die Erstposition in den Satz integrierte Topik wird aber im Ungarischen nicht fokussiert.

(36) A DEficit, az NEM egy offshore-cégnél landolt?

(37) A deficit NEM egy offshore-cégnél landolt?

\section{V2-Entscheidungsinterrogativsätze im Deutschen}

V2-Entscheidungsinterrogativsätze werden im Deutschen konkurrierend markiert: Durch die Verb-zweit-Stellung ähneln sie den Deklarativsätzen, durch die satzabschließende steigende Intonation den Interrogativsätzen. Altmann zählt sie zu den Interrogativsätzen, allerdings mit der Einschränkung, dass mit ihnen so genannte assertive Fragen realisiert werden, mit denen der Sprecher einen Sachverhalt assertiert, den er stark, aber doch nicht mit absoluter Sicherheit annimmt und am Ende deshalb doch in Frage stellt. Bei Brandt et al. (1992) werden sie als Deklarativsätze eingestuft, mit denen auf der Ebene der Pragmatik eine Frage realisiert wird. Die satzabschlieBende Intonation sei in diesem Konzept das Merkmal der pragmatischen Funktion. Gunlogson (2003) sieht die Hauptfunktion dieses Satztyps darin, dass mit der satzeröffnenden nicht verbalen Konstituente auf einen common ground, auf eine gemeinsame Basis des Senders und des Empfängers Bezug genommen wird. Ich betrachte diese Sätze als Interrogativsätze, die keine (oder mindestens nicht unbedingt eine) assertive Funktion haben. In Anlehnung an den Ansatz von Gunlogson wird im Folgenden mit ausgewählten Belegen dafür argumentiert, dass die Verwendung der V2-Entscheidungsinterrogativsätze im Deutschen auch ähnliche kontextuelle bzw. situative Gründe haben kann wie im Ungarischen, indem mit dem anfänglichen Konstituente eine gemeinsame Basis als Grundlage für die Fragestellung festgelegt wird. 
Am häufigsten kommen textkonnektierende Adverbien, Konjunktionaladverbien bzw. vokativisch zu interpretierende Nominalphrasen im Vorfeld des Entscheidungsinterrogativsatzes vor. Sie sind auch im Deutschen sogar in öffentlichen, stark normierten Situationen durchaus erlaubt. Mit dem Vorfeldelement wird hier eindeutig auf die gemeinsame Basis Bezug genommen.

\section{Deswegen machen Sie beim Verbot von Killerspielen nicht mit? [Zwischenruf] \\ Und das Fräulein war also wieder einmal am Fluss? [Dramentext]}

In lockeren Alltagsgesprächen habe ich ferner sporadisch auch im Deutschen Belege für V2-Entscheidungsinterrogativsätze gefunden, in denen eine referierende Phrase im Vorfeld auf einen ähnlichen common ground verweist wie im Ungarischen.

(40) - ich hab mir jetzt ein ganz großes Band gekauft (.) so strech ne nich samt ein haarband

- und das ziebst dann an? [Gespräch]

(41) - ja (.) stehst da vorm manhatten am 1. dezember (.) so richtig schön arschkalt (.) die straßen arschglatt (.) ja doll [...] zehn meter vor der schikane ne vollbremsung machen (.) also obne daß die räder blockieren [...]

- das machen die so? [Gespräch]

(42) - und denn aufm weg nach hause wir gingen zurückauf einmal stellt sich da son passat quer auf die straße die steigens aus musst ich zurück kriminalpolizei habn $n$ marke gezeigt und (.) habn mir die waffe abgenommen aber sonst nix gesagt

- mbm keine anzeige hast du gekriegt? [Gespräch]

In Beispiel (40) weist das ins Vorfeld gestellte Pronomen auf das Haarband zurück, auf den gemeinsamen Hintergrund, in dessen Zusammenhang die Frage gestellt wird. In (41) handelt es sich allgemein um bestimmte Möglichkeiten, was bestimmte Autofahrer in Amerika machen können. Die Frage bezieht sich darauf, ob diese nur Möglichkeiten darstellen, oder ob die Vollbremsung wirklich so vollzogen wird. Mit dem Pronomen im Vorfeld wird die unmittelbare Verbindung mit der vorangehenden Äußerung des Partners hergestellt.

Sogar eine Nominalphrase ist in dieser Funktion belegt (42). Der Ausdruck keine An₹eige ist deshalb ein angemessener Ausgangspunkt für eine Fragestellung, weil eine Anzeige wegen unerlaubten Waffenbesitzes - wie allgemein bekannt - sehr wahrscheinlich wäre.

Eine komplexe Interpretation erlaubt die als Beispiel (43) dienende Broschüre. 
(43)

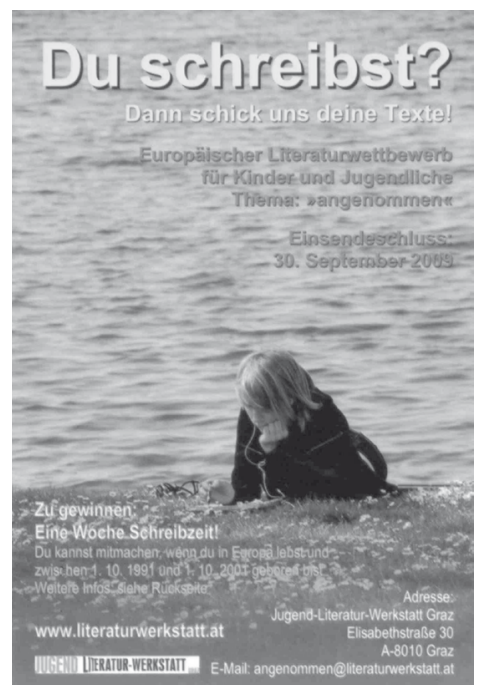

Mit dem im Vorfeld stehenden Pronomen $d u$ wird auf das Bild bzw. auf den Leser Bezug genommen. Da der Leser zugleich sieht, dass ein Junge im Gras liegt und schreibt, wäre eine Frage wie Schreibst du? nicht sinnvoll zu interpretieren. Die Feststellung Du schreibst. hätte aus dem gleichen Grund auch keine sinnvolle Interpretation. Es geht um eine wirkliche Frage, mit dem besetzten Vorfeld wird unmittelbar auf die Situation Bezug genommen. Das Verb schreiben kann hier nicht im Sinne von ,Buchstaben auf ein Papier setzen' verstanden werden, denn das sieht man ja, sondern in dem Sinne, in dem Schriftsteller oder Journalisten schreiben: in schriftlicher Form etwas Künstlerisches hervorbringen. Das kann man nämlich auf dem Bild optisch nicht wahrnehmen. Die unmittelbare Bezugnahme auf die Situation mit Hilfe des im Vorfeld stehenden Pronomens deutet auf die Notwendigkeit der komplexen Interpretation hin, indem Text und Bild eng aufeinander bezogen interpretiert werden müssen. Insgesamt ist es im Deutschen mindestens in besonderen Situationen möglich, Phrasen ins Vorfeld zu stellen, die der kontextuellen bzw. der situationellen Einbettung dienen und auf den gemeinsamen Hintergrund, auf den common ground für die Fragestellung verweisen.

Freilich ist diese Möglichkeit in anspruchsvollen öffentlichen Situationen, besonders in solchen, in denen der Sprachgebrauch eng an die schriftsprachliche Norm gebunden wird, nicht gegeben. In Bundestagsdebatten habe ich kein einziges Beispiel mit einer referierenden Phrase im Vorfeld des Entscheidungsinterrogativsatzes gefunden, das nicht als assertive oder als Echo-Frage interpretiert werden sollte. Ich habe deshalb im Folgenden gezielt nach Kompensationsmöglichkeiten für die vorliegende Funktion 
gesucht. Die meist verwendete Strategie der deutschen Sprecher besteht darin, die als Grundlage für die Fragestellung dienende festzuhaltende Information in einem getrennten, vorangestellten Satz auszuführen und die eigentliche Frage dann mit einem V1-Satz zu formulieren. In (45) wird sogar explizit festgehalten, dass der erste Satz als der allgemein bekannte Hintergrund für die Fragestellung dient.

(44) Die Fabrt kostet bis zum dritten Kind pro Kind 23 Euro und für das vierte, fünfte, sechste und jedes weitere Kind kostet die Fahrt 38 Euro. Halten Sie das für familienpolitisch vertretbar? [Debatten]

$\rightarrow$ Die hohen Fahrtkosten für Kinder - halten Sie die für familienpolitisch vertretbar?

(45) Vor dem Hintergrund, dass bereits im August des letaten Jahres unter anderem in der „FAZ“ auf Hinweise rekurriert wurde, dass unter Umständen in Nordkorea, im Irak [...] Biokampfstoffe und entsprechende gefährliche Erreger existieren [...], frage ich Sie: Ist es richtig, dass die allgemeinen Gefährdungshinweise bis heute allgemein geblieben sind? [Debatten]

$\rightarrow$ Was die Biokampfstoffe anbelangt, ist es richtig, dass ...

Ein wesentlicher Motivationsfaktor für derartige Strukturen ist natürlich, wenn die hintergründige Information an sich so komplex ist, dass sie nur in einem getrennten Satz ausgeführt werden kann. Es gibt jedoch auch ausreichend Beispiele für wesentlich weniger komplexe Hintergrundinformationen, die in einem vorangestellten Satz realisiert werden. Sporadisch sind solche Strukturen auch in den anderen Teilkorpora belegt.

(46) Es wird von einer Anzabl von 10000 Betrieben gesprochen. Teilen Sie diese Ansicht? [Debatten]

$\rightarrow$ Die Ansicht über die 10000 Betriebe - teilen Sie sie?

(47) für alle (.) liest du das vor? [Gespräche]

$\rightarrow$ Den für alle geschriebenen Text - liest du ihn vor?

\section{Fazit}

1) In beiden Sprachen wird die lineare Satzstruktur grundsätzlich durch den Verbalkomplex bestimmt.

2) Die V2-V1-Opposition ist im Deutschen ziemlich fest an Satztypen gebunden, es gibt aber markierte V1-Deklarativ- und auch V2-Entscheidungsinterrogativsätze.

3) Die Vorfeldbesetzung dient im V2-Entscheidungsinterrogativsatz vor allem zur Herstellung des unmittelbaren Situationsbezugs bzw. der Festlegung eines gemeinsamen Hintergrundes. 
4) Im Ungarischen stehen VK2- und VK1-Sätze in einem ähnlichen Verhältnis zueinander.

5) V2-Entscheidungsinterrogativsätze sind im Deutschen in strenger normierten Situationen nur in besonderen Fällen zu benutzen, damit erklärt sich auch ihre relativ geringe Frequenz, während VK2-Entscheidungsinterrogativsätze im Ungarischen uneingeschränkt möglich sind. Im Deutschen wird der common ground, der gemeinsame Hintergrund für die Fragestellung oft im Vortext, in den vorangehenden Sätzen festgelegt.

\section{Anhang}

Das zugrunde liegende deutsch-ungarische Vergleichskorpus:

\begin{tabular}{|c|c|c|c|}
\hline \multicolumn{2}{|c|}{ Deutsche Texte } & \multicolumn{2}{|c|}{ Ungarische Texte } \\
\hline Teilkorpus & Umfang & Teilkorpus & Umfang \\
\hline $\begin{array}{l}\text { Plenarsitzungsprotokolle } \\
\text { des Deutschen Bundes- } \\
\text { tags aus dem Jahr } 2003 \\
\text { Debatten/Zwischenrufe }\end{array}$ & $\begin{array}{l}3.887 .145 \\
\text { Textwörter }\end{array}$ & $\begin{array}{l}\text { Plenarsitzungsprotokolle } \\
\text { des Ungarischen Land- } \\
\text { tags aus dem Jahr } 2000 \\
\text { Debatten/Zwischenrufe }\end{array}$ & $\begin{array}{l}4.492 .912 \\
\text { Textwörter }\end{array}$ \\
\hline $\begin{array}{l}\text { Gespräche von Schülern } \\
\text { in einem Schülerheim } \\
\text { (vgl. Schlobinski/Kohl/ } \\
\text { Ludewigt 1998) }\end{array}$ & $\begin{array}{l}85.866 \\
\text { Textwörter }\end{array}$ & $\begin{array}{l}\text { Gespräche von Studenten } \\
\text { in einem Studentenheim } \\
\text { (vgl. Keszler 1983) }\end{array}$ & $\begin{array}{c}34.917 \\
\text { Textwörter }\end{array}$ \\
\hline $\begin{array}{l}4 \text { Dramentexte aus dem } \\
\text { 20. Jahrhundert }\end{array}$ & $\begin{array}{c}42.339 \\
\text { Textwörter }\end{array}$ & $\begin{array}{l}9 \text { Dramentexte aus dem } \\
\text { 20. Jahrhundert }\end{array}$ & $\begin{array}{l}156.846 \\
\text { Textwörter }\end{array}$ \\
\hline
\end{tabular}

\section{Literatur}

Admoni, Wladimir (1990): Historische Syntax des Deutschen. Tübingen.

Ágel, Vilmos/Hennig, Mathilde (2006): Theorie des Nähe- und Distanzsprechens. In: Ágel, Vilmos/Hennig, Mathilde (Hg.): Grammatik aus Nähe und Distanz. Theorie und Praxis am Beispiel von Nähetexten 1650-2000. Tübingen, S. 3-31.

Altmann, Hans (1993a): Satzmodus. In: Jacobs, Joachim et al. (Hg.): Syntax. Ein internationales Handbuch zeitgenössischer Forschung. 1. Halbbd. (= Handbücher zur Sprach- und Kommunikationswissenschaft 9.1). Berlin/New York, S. 1006-1029.

Altmann, Hans (1993b): Fokus-Hintergrund-Gliederung und Satzmodus. In: Reis, Marga/Rosengren, Inger (Hg.): Fragesätze und Fragen. (= Linguistische Arbeiten 257). Tübingen, S. 1-37. 
Altmann, Hans (2009): Cleft- und Pseudocleft-Sätze (Spalt- und Sperrsätze) im Deutschen. In: Brdar-Szabó, Rita/Knipf-Komlósi, Elisabeth/Péteri, Attila (Hg.): An der Grenze zwischen Grammatik und Pragmatik. (= Deutsche Sprachwissenschaft international 3). Frankfurt a.M. u.a., S. 13-34.

Bassola, Peter (2001): Wortstellung Deutsch-Ungarisch. In: Bassola, Peter et al. (Hg.): Wortstellung im Sprachvergleich (deutsch - niederländisch - polnisch - ungarisch). (= Deutsch im Kontrast 20). Tübingen, S. 9-62.

Brandt, Margareta et al. (1992): Satztyp, Satzmodus und Illokution. In: Rosengren, Inger (Hg.): Satz und Illokution. Bd. 1. (= Linguistische Arbeiten 278). Tübingen, S. $1-90$.

É.Kiss, Katalin (2002): The syntax of Hungarian. Cambridge.

É.Kiss, Katalin (2007): Az ige utáni szabad szórend magyarázata [Erklärung der freien Wortstellung hinter dem Verb]. In: Nyelvtudományi Közlemények [Linguistische Veröffentlichungen] 104, S. 124-152.

É.Kiss, Katalin/Kiefer, Ferenc/Siptár, Péter (1998): Új magyar nyelvtan [Neue ungarische Grammatik]. Budapest.

Gunlogson, Christine (2003): True to form: rising and falling declaratives as questions in English. New York u.a.

Kenesei, István/Vago, Robert M./Fenyvesi, Anna (1998): Hungarian. London u.a.

Keszler, Borbála (1983): Kötetlen beszélgetések mondat- és szövegtani vizsgálata. [Die syntaktische und textlinguistische Untersuchung lockerer Gespräche]. In: Rácz, Endre/Szathmári, István (Hg.): Tanulmányok a mai magyar nyelv szövegtana köréből. [Studien aus dem Bereich der Textlinguistik der ungarischen Gegenwartssprache.] Budapest, S. 164-202.

Keszler, Borbála/Lengyel, Klára (2008): Ungarische Grammatik. Hamburg.

Kiefer, Ferenc (Hg.) (1992): Strukturális magyar nyelvtan. Bd. I: Mondattan [Strukturelle ungarische Grammatik. Bd. I: Syntax]. Budapest.

Kiss, Jenő (2003): Ungarisch. In: Roelcke, Thorsten (Hg.): Variationstypologie. Variation Typology. Ein sprachtypologisches Handbuch der europäischen Sprachen in Geschichte und Gegenwart. Berlin/New York, S. 905-918.

Kiss, Jenő/Pusztai, Ferenc (2003): Magyar nyelvtörténet [Ungarische Sprachgeschichte]. Budapest.

Koch, Peter/Österreicher, Wulf (1985): Sprache der Nähe - Sprache der Distanz. Mündlichkeit und Schriftlichkeit im Spannungsfeld von Sprachtheorie und Sprachgebrauch. In: Romanistisches Jahrbuch 36. Berlin/New York, S. 15-43.

Komlósy, András (1992): Régensek és vonzatok [Regentien und Dependentien]. In: Kiefer (Hg.), S. 299-527.

Önnerfors, Olaf (1997): Verb-erst-Deklarativsätze. Grammatik und Pragmatik. (= Lunder germanistische Forschungen 60). Stockholm.

Schlobinski, Peter/Kohl, Gabi/Ludewigt, Irmgard (1998): Jugendspezifische Sprechweisen. CD-ROM. (= Text- und Tonbibliothek sprachlicher Varietäten 02). München. 
Tompa, József (1968): Ungarische Grammatik. Budapest/Den Haag.

Zifonun, Gisela/Hoffmann, Ludger/Strecker, Bruno (1997): Grammatik der deutschen Sprache. 3 Bde. (= Schriften des Instituts für Deutsche Sprache 7). Berlin/ New York. 